OAI-PMH: http://www.indteca.com/ojs/index.php/Revista Scientific/oai

Artículo Original / Original Article

\title{
Mirada Axiológica al Discurso Formativo del Profesional de Enfermería
}

\author{
Autoras: Carol Del Carmen Terán González \\ Universidad Nacional Experimental "Rafael María Baralt", UNERMB \\ carolteranula@gmail.com \\ Trujillo, Venezuela \\ Yurquelis Rico Chacón \\ Universidad Nacional Experimental "Rafael María Baralt”, UNERMB \\ yurquelisricoch@gmail.com \\ Táchira, Venezuela
}

\section{Resumen}

Este estudio buscó aproximarse a la realidad teórico axiológico del discurso formativo del profesional de enfermería desde su contexto social. Para ello, se partió de ciertos apartados y procesos dentro de la investigación la cual tuvo como propósito: Interpretar los discursos del docente en la formación del profesional de enfermería desde los elementos axiológicos que se presentan en la praxis social en la Universidad de los Andes, Trujillo, Venezuela. La explicación que requirió la trascendencia de esta investigación tuvo su basamento desde la relación e interacción entre el investigador y lo investigado, donde se establecieron representaciones para construir un modelo teórico a partir de la realidad teórico axiológico del discurso formativo del profesional de enfermería desde su contexto social. En cuanto a la argumentación teórica, se desarrolló como unidad temática desde los componentes axiológicos y el discurso de formación del profesional de enfermería. Para su metodología, se argumentó desde la hermenéutica, y se presentaron elementos interpretativos desde la formación axiológica del enfermero y desde el espacio en que este profesional converge. Se llegó a la conclusión que enfoque axiológico destaca el carácter valorativo de la ciencia de la salud, en este caso, enfermería; partiendo de conceptos elementales y del perfil humanista de esta ciencia. Por ende, más allá de esto poder enfatizar el pensamiento valorativo en el profesional de enfermería, a partir de la formación de valores en educandos, futuros profesionales capaces de actuar y evaluar una situación determinada del paciente, sustentados en los principios humanos y éticos.

Palabras clave: discurso; formación; profesional. 


\title{
Axiological Look to the Discourse of the Nursing Professional Training
}

\begin{abstract}
This study tried to come closer to the theoretical axiological reality of the formative speech of the professional of infirmary from his social context. In order to that, it took into account certain paragraphs and processes inside the investigation which had as intention: Interpreting the speeches of the teacher in the formation of the professional of infirmary from the axiological elements that appear in the social practice in the University of the Andes, Trujillo, Venezuela. The explanation that needed the transcendence of this investigation had its basement from the relation and interaction between the investigator and the investigated, where representations were established to construct a theoretical model from the theoretical axiological reality of the formative speech of the professional of infirmary from his social context. As for the theoretical argumentation, it developed as thematic unit from the axiological components and the speech of formation of the professional of infirmary. For its methodology, it was argued from the hermeneutics, and interpretive elements were presented from the axiological formation of the nurse and from the space on which this professional converges. It came near to the conclusion that focuses axiological the character distinguishes value from the science of the health, in this case, infirmary; departing from elementary concepts and from the humanist profile of this science. For end, beyond this to be able to emphasize the thought value in the professional of infirmary, from the formation of values in pupils, future professionals capable of acting and evaluating a certain situation of the patient, sustained in the human and ethical principles.

Keywords: speech; training; professional.




\section{Introducción}

El explorar la realidad del discurso del docente universitario, implica escudriñar sobre la labor y formación que se desempeña en el estudiante de la carrera profesionalización en enfermería en la facultad de medicina, escuela de enfermería en la Universidad de los Andes estado Trujillo, por tal motivo las investigadoras realizarán una búsqueda exhaustiva de teorías que fundamenten la situación del objeto de estudio y por tanto, su ontología.

El escenario en estudio, tiene como tema de enfoque el discurso de los docente en la formación de profesionales de enfermería y su relación con la ética y valores, es decir, la parte axiológica; sin embargo el entorno investigado es la Universidad de los Andes que según la reseña histórica en línea la misma, plantea que tuvo su origen en el año 1785, cuando Fray Juan Ramos de Lora, primer obispo de Mérida, fundó en esta ciudad una casa de educación para los jóvenes con vocación por la carrera sacerdotal; en ella se les impartiría clases de religión, lengua latina y moral. El 21 de septiembre de 1810 la Junta Superior que gobernaba en Mérida le otorgó al Seminario el ansiado Título de "Real Universidad de San Buenaventura de Mérida de los Caballeros".

Posteriormente, el 24 de septiembre de 1883, estando de nuevo Guzmán Blanco en la presidencia de la República, ordena la venta de todos los bienes de la Universidad de Mérida, con lo cual esta se arruinó y pasó a depender completamente del presupuesto del estado. Por consiguiente, en el año de 1905 se le restituyó su nombre. Es a partir de esa fecha, cuando definitivamente retomó su denominación Universidad de Los Andes; la misma, es una universidad nacional autónoma, financiada por el estado. Comprende un área de $360.719 \mathrm{~m} 2$, distribuidos a lo largo de los tres estados Andinos: Mérida, Táchira y Trujillo además de las extensiones de la Facultad de Medicina que se encuentran en las ciudades de Guanare, Valle de La Pascua y Barinas.

Hoy en día y a través de los datos emanados de la página principal de 
la Universidad de los Andes, la escuela posee dos programas de formación estudiantil: el Regular con duración de 10 semestres presenciales dirigidos a los estudiantes quienes egresan de la educación media y diversificada. Y el de Profesionalización o Prosecución de Estudios de Técnicos Superiores Universitarios con una duración de 3 semestres. Este segundo programa se sustenta en las premisas de la educación andragógica, las técnicas de la educación a distancia y el estudio-trabajo del participante, donde no se requiere la relación permanente y continua en los establecimientos universitarios; aspectos que lo ubican en un sistema educativo innovador. Hoy en día, este programa se desarrolla en Mérida, como sede principal y posee extensiones en Barinas, Táchira, Valera, Guanare, Lara, Apure y el Zulia.

Por ende, en el estado Trujillo en la ciudad de Valera, existe una de estas sedes donde se profesionaliza el personal de enfermería, es decir, licenciatura en enfermería; allí es donde se origina este pensamiento, debido a que la formación que imparten los docentes universitarios no concuerda con lo deseado en el ámbito práctico del enfermero (a), todo esto referente a lo axiológico. Ya que la axiología tiene que ver con los valores y ética; valores que son enseñados desde la crianza en sus respectivos hogares, influyendo de manera importante desde la educación inicial hasta la universitaria, ya que el ser humano como sociedad se forma y fortalece con cultura, valores, experiencias vividas, según su origen, entre otros tipos de situaciones y entornos que influyen significativamente en el proceso de desarrollo de una persona.

Se puede decir, que el enfoque axiológico trata de estudiar la instrucción del ser desde su nacimiento a través de la educación; por eso la educación familiar tiene mucho que ver, pues la relación y el inculcar valores adecuados fortalecerá la personalidad de cada ser. Debido a esto, el instruir e integrar valores y aprendizajes de manera tal que se formen mujeres y hombres de bien, con personalidades que generen un cambio productivo y cortes en la 
sociedad, con respeto, solidaridad, responsabilidad, igualdad, verdad, entre otros. Y allí es donde participa la educación, pues de este modo se atienden las necesidades de cada individuo, allí es cuando se conforta el estudiante, y así transformar en fortalezas y habilidades sus debilidades. De igual modo, la universidad refleja lo universal, es decir, el todo o lo abierto por descubrir, es por eso que el estudiante universitario se prepara para organizar y proporcionar un mejor profesional a la comunidad.

Pese a que, en la actualidad esta situación se visualiza de manera diferente, pues existe una sustitución de valores que alteran la praxis universitaria, en el caso del profesional de enfermería ha existido una deficiencia irrevocable en el desarrollo de estos valores como la ética, la moral, igualdad, solidaridad, respeto, responsabilidad, entre otros. $Y$ estas circunstancias se evidencian al momento de su desempeño laboral tanto en centros asistenciales públicos como privados, donde la discriminación va por delante, el diferenciar la situación económica de cada uno, si es de escasos recursos o no; cabe decir que el personal de enfermería se distingue por sus funciones asistenciales, administrativas, gerenciales y comunitarias, donde el cien por ciento de su atención va dedicado a un usuario o paciente.

Partiendo de lo anterior Morín (1999), plantea:

Existe un conocimiento que es un comprehensivo y que se basa en la comunicación, la empatía, inclusive la simpatía, intersubjetivas. Así comprendo las lágrimas, la sonrisa, la risa, el miedo, la ira, cuando veo al alter ego como alter ego, cuando puedo experimentar los mismos sentimientos que él. Comprender conlleva un proceso de identificación y de proyección de sujeto a sujeto. (pág. 99).

Se puede decir, entonces que el enfermero (a) debe estar formado con alto sentido ético, capacitado para el desempeño eficiente de las funciones y tareas propias de Enfermería General. Su ejercicio está orientado a la promoción, conservación y restitución de la salud individual y colectiva a nivel 
de establecimientos y de programas de salud intra y extra institucional. La asistencia en enfermería, es destacada por ser en el ámbito practico donde desempeña su rol esencial, el de ayudar y facilitar la atención necesaria a un paciente; es decir, brindarle cuidados directos, servicios de salud adecuados.

\subsection{Discurso Docente en la realidad Universitaria}

En la actualidad, la realidad universitaria consta de una formación pertinente y permanente, ya que forja a la creación de un nuevo ciudadano, profesional y adulto productivo; a través de la educación constante y a la vanguardia con otros países. Pues la realidad educativa ha obtenido un cambio que genera transformación en la sociedad, mas, sin embargo, en muchas ocasiones no es la esperada, la educación universitaria se diferencia por ser más complementaria, por incentivar y demostrar la innovación educativa de cada participante y profesional, como la del docente formador.

El discurso docente, se enfoca en lo que observa y desarrolla con el estudiante o participante, lo que implica esa relación profesor - estudiante, la enseñanza que se transmite y el aprendizaje que se recibe; pues bien, la universidad refleja lo universal, un todo en definido ámbito, es decir, una carrera o profesionalización. El docente universitario aplica estrategias de enseñanza que generan lo deseado, el cambio en el pensamiento del estudiante, la transformación del cómo ser pensante, como comunidad y sociedad, proporcionando el reto universitario el cual es la productividad y desarrollo en general.

De esta manera, es como la sociedad del conocimiento presenta una conciencia histórica, cultural, política e ideológica pasando a incorporarse al Sistema Educativo. Es por eso, que el desarrollo de un país radica en el grado de educación y formación de sus docentes; por ello, la educación es concebida como la suprema contribución al futuro del mundo actual. Referente al docente universitario, deben ser escuchados sus puntos de vista, percepciones, 
interpretaciones, vivencias de su mundo cotidiano de práctica pedagógica y andragógica, entonces así realizar una interpretación intersubjetiva donde se refleje una visión concreta de la realidad, donde se observe, diagnostique y evalúe la práctica educativa, tal cual como esta se presenta.

Acorde a esto, el discurso del docente se asume como una estrategia formada no sólo por palabras y expresiones, sino por valores e ideología que influyen de manera directa en la labor educativa del facilitador, y en la respuesta del participante. Ricoeur (1995:394), añade el discurso que recurre de manera deliberada, a la metáfora viva para obtener significaciones nuevas de la impertinencia semántica y dar a conocer nuevos aspectos de la realidad mediante la innovación semántica. Significa entonces que ese fenómeno llamado discurso, es un informante clave de lo que sucede en la actividad educativa y lo que ocurrirá después de ella, es una herramienta elemental del proceso educativo, con una elevada incidencia en el comportamiento de los estudiantes, ya que su accionar transforma desde la significación del hilo discursivo.

En consecuencia, hay que redescubrir el valor ético del discurso del docente universitario y de tal modo ir tras la complejidad del discurso pedagógico, se hace necesario partir de los ambientes educativos donde docentes y estudiantes se encuentran a diario y de acuerdo con el paradigma de vida que manejen, conjugarán esfuerzos o marcarán distancia entre uno y otro. Con el fin de interpretar el discurso docente en la realidad universitaria, a través del ámbito educativo, y la proporción que este enmarcando en la sociedad, como fundamento primordial al ser cambiante y productivo, participe de generar innovación en la educación universal.

\subsection{Propósito de la investigación}

Interpretar los discursos del docente en la formación del profesional de enfermería desde los elementos axiológicos que se presentan en la praxis 
social en la Universidad de los Andes, Trujillo, Venezuela.

\section{Recorrido Teórico}

En este momento de la investigación, se acude a la temática y a cada una de las categorías o dimensiones que sustentaron los planteados teóricos. Esto a consecuencia de que existe un infinito en conocimiento, y detalles diferentes desde el punto discursivo y formativo. Bien, estas teorías han sido una luz e incentivo en el camino emprendido por la autora sobre el discurso, así como la savia del conocimiento, definida en las cinco categorías desplegadas, es decir, lo ético, lo axiológico, lo social, lo andragógico y el poder.

\subsection{Discurso}

La palabra discurso se deriva del latín discursus, el discurso es un testimonio o exposición de pensamientos; es decir, un mensaje que se manifiesta de manera pública o bien sea privada. De tal modo, el discurso es la forma de expresar palabras ante un grupo de personas o una persona, para transmitir un alegato o pensamiento ante una situación; el mismo trata de lo que considera o piensa cada persona como ser pensante de un tema, indiferentemente la ideología, cultura, religión, entre otros, donde no se compartan las mismas creencias, pero se hace la diferencia del discurso de cada uno en referencia a la realidad de la persona.

El mismo, es un medio de comunicación cuyo objetivo es explicar o transmitir algún tipo de información. En un sentido más amplio, se pudo precisar el discurso como todo proceso de expresiones amplias 0 fragmentadas que sirvan para enunciar en términos o palabras. Van Dijk (1999a): esboza, el discurso es poder y la persuasión es el mayor controlador

de actos lingüísticos en la modernidad. Esto se refiere, a que todo discurso busca crear una intención en el otro o un fin, el cual recibe el mensaje; el 
mismo está cargado de imágenes, símbolos, sentidos que causan una reacción en quien lo percibe. De este modo, el discurso genera un cuento, una travesía, donde se imagina lo que se están escuchando y redactando y así comprendiendo el objetivo del emisor.

También las autoras consideran el discurso, como parte de la interacción social, ese encuentro entre culturas, pensamientos, creencias, ideologías, entre otros; donde se llega a un consenso entre la información y lo que se discurre; por eso lo caracteriza Van Dijk (1999b), citando a Fairclough y Wodak (1994): como una forma de acción social. Donde se demuestra que la sociedad es participe esencial en la relación y desenvolvimiento con el discurso indiferentemente de su índole.

Ahora bien, una vez puntualizado el acercamiento a la definición de discurso es importante señalar que el mismo se nutre dentro de su alegato discursivo dentro de lo axiológico, lo ético, lo andragógico, lo social y el poder, bajo el contexto de la formación universitaria. Entonces, es a través del discurso y los enunciados antes mencionados que se alimenta el futuro profesional de enfermería en los distintos campos laborales.

\subsection{Discurso Axiológico}

Dentro de este estudio al hablar de discurso formativo, hago referencia a los enunciados, alegatos y fundamentos discursivos empleados en el aula por parte del docente en las distintas áreas del conocimiento, en el caso de la investigación, el profesor universitario formador del profesional de enfermería. Por tal motivo el lenguaje, habla o discurso docente, como herramienta educativa y formadora puede favorecer el aumento intelectual, emocional, moral o social del educando, según los objetivos que persiga. El empleo del discurso en el aula, en gran parte genera diversas particularidades de relación profesor - estudiante.

Lo anterior quiere decir, que el discurso formativo del docente ocasiona 
un impacto en el alumno. Según Martínez y Pérez (s.f.), el discurso del docente en el aula tiene una semiótica, la cual se deriva de dimensiones o funciones, como la instructiva la cual señala el conocimiento y dominio de la asignatura o temática del profesor universitario, también la dimensión afectiva y motivacional en la cual influye el discurso de manera subjetiva e intersubjetiva, pues hay expresión de estados de ánimo o palabras afectivas y de estímulo. Además de la dimensión social y ética, las cuales hay que resaltar debido a su atribución en la acción forjadora del estudiante, en lo que respecta al desarrollo personal y la realidad en la comunidad.

El tipo de rol del docente, también interviene en el discurso formativo ya que argumenta que posición esta toma ante una situación o tema; pues protagoniza las herramientas a utilizar y desarrollar en su área; esto representa el pensamiento del docente y su forma de debatirlas. Es decir, el discurso instructivo es un instrumento clave para la comprensión y mejora de la calidad educativa, por tanto, la construcción de la realidad personal como formación humana se explica, a través del discurso educativo y la intervención que el mismo produce.

Cabe señalar que el discurso en general, y el discurso educativo específicamente, no son una simple representación del pensamiento en el lenguaje, sino que ha de ser tratado como un modo social de pensar (Mercer, 1996:94). Por ello, el discurso educativo es el responsable no sólo de la construcción del conocimiento en el ámbito de estudio, sino también de la propia realidad en la comunidad como parte de la interacción social.

\subsection{El discurso ético en la formación del profesional de enfermería}

Se puede decir, que con lo anterior definido con respecto al discurso ético, se tiene una noción o idea de lo que representa el hablar con ética y moral; pues el dialogo genera respeto, propiedad, creencia en lo que se está transmitiendo. De este modo, el discurso ético en la formación del profesional 
de enfermería se ve reflejado cuando se está instruyendo al estudiante de enfermería, en su vocación, desempeño y desarrollo cognitivo; es decir, la educación que se imparte a través de la charla, fomentada en valores que originen un desarrollo personal y profesional en el estudiante.

Por eso, la enfermería se caracteriza por ser ética y humanista, por el trato directo con su profesión y praxis social, pues el enfermero(a) transmite empatía, respeto, responsabilidad, entre otros valores que discurren a una ética profesional. Sin embargo, esta situación no sucede, pues el discurso ético formativo no es el que se quiere en la realidad y actualidad, ya que no es forjado y no emerge como debe ser, es a través de ese discurso, que se debe ocasionar la transformación de pensamientos y actitudes, como índole o referencia en la salud y así demostrar lo característico que es el profesional en enfermería.

\section{Representación ontoepistémica y metodológica de la investigación}

El abordaje metodológico concreto de esta investigación remite la necesidad de identificar los discursos formativos, pedagógicos y de reinserción desde los diferentes contextos del informante que le permitirá a la autora aproximarse al sentido del estudio, intentando ubicar la diversidad de perspectivas de los actores investigados. De esta manera, se introdujo en las fuentes relevantes del estudio: el discurso formativo y sus referentes como el discurso axiológico, de poder, social, andragógico y ético en el profesional de enfermería de la Universidad de los Andes, Valera, Estado Trujillo; para luego, con esta recogida de información abordar la interpretación; entonces aquí se tratará de captar el discernimiento de la experiencia, desde las categorías significativas se irán ubicando los relatos, hasta descubrir el proceso vivido y real.

Haciendo notable, que a los docentes se les investigó desde sus experiencias en la formación de profesionales de enfermería, la forma cómo 
enuncian los discursos en su praxis educativa, para luego comprender los significados que le daban a lo habitual durante la formación de estos estudiantes; y así contribuir en la realidad educativa y por ende social, todo esto a partir del discurso docente y sus diferentes modos de uso.

Al bosquejar la hermenéutica, Ibáñez (2005): sustenta que todo saber halla sus contextos de inteligibilidad en el círculo hermenéutico. Considerando lo diferido, la esencia no se construye por el simple hecho de anexar elementos discretos, sino que supone un constante movimiento y consenso desde lo íntegro y total del texto.

Por tal razón, cada uno de los distintos elementos favorecen en la construcción del significado global, y cada uno logra a su vez su sentido en función de ese significado integral. Es decir, nada adquiere sentido si no es a través de su incorporación a un marco interpretativo, que determina en parte el posible sentido de cualquier elemento parcial, aunado a sus condiciones sociohistóricas.

Es desde estas premisas, donde se adquiere la importancia y relevancia discursiva de los docentes, con relación a su expresión en la práctica educativa diaria, tomando en cuenta el conjunto de elementos de parten de su propia realidad social y educativa para converger en un interés total.

Figura 1. Circulo hermenéutico. Teorías.

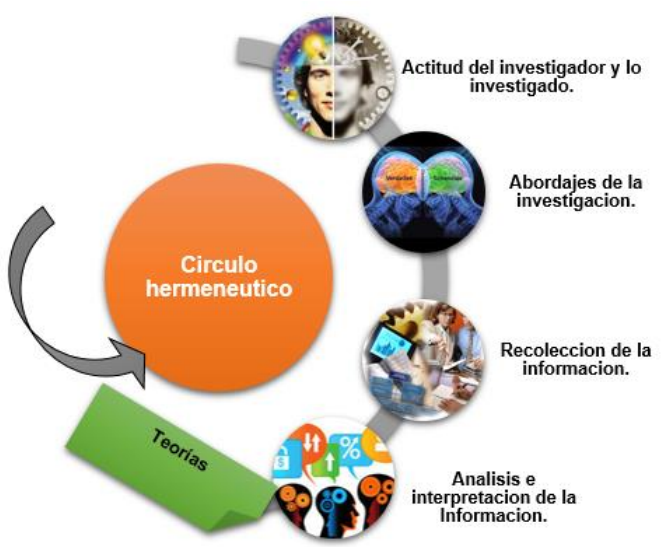

Fuente: Las Autoras (2016). 
Finalmente, puede observarse en el esquema mental presentado anteriormente y llamado "Circulo Hermenéutico", donde se proyecta el proceso de investigación ya descrito desde el método hermenéutico y sus principales abordajes. El cuál es la mirada de las autoras para el desarrollo del momento metodológico y ontoepistémico de la investigación en curso.

Figura 2. Abordajes de investigación. Epistemológico, Axiológico, Ontológico y Metodológico.

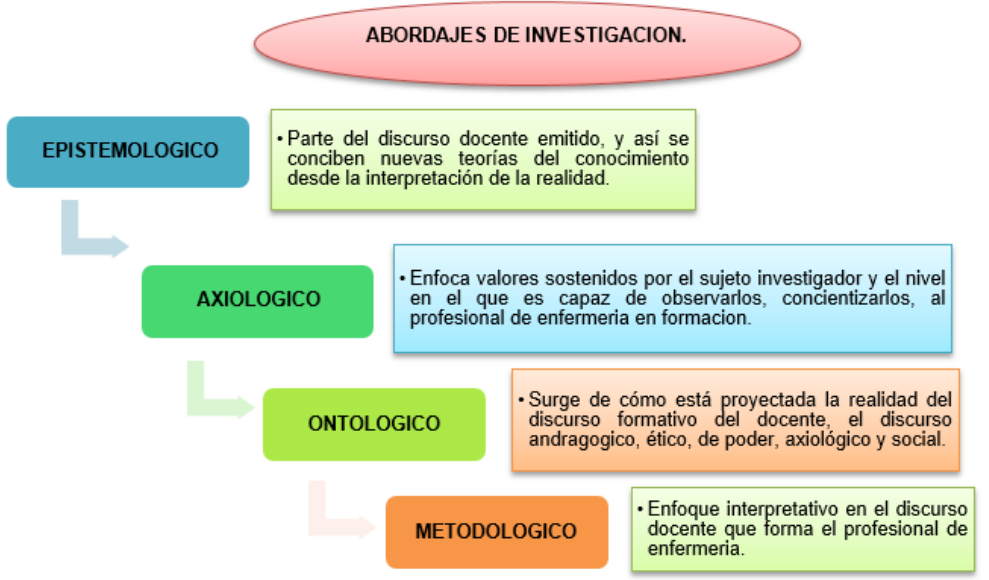

Fuente: Las Autoras (2016).

\section{A manera de Conclusión}

El profesional de enfermería, se destaca por ser personas capacitadas y competentes al momento de dar un servicio de salud; ya que su misión es resguardar la vida del ser humano, promocionar la salud y bienestar físico y mental de todas y todos, además de atender en enfermedades o hasta la muerte a un ser que necesite o amerite cuidados propios de enfermería, es decir la función de enfermería es la de ser: Sustituta: reemplaza lo que le falta al paciente, Ayudante: brinda el cuidado clínico, y Compañera: fomenta la relación terapéutica o transpersonal. Con lo anterior dicho, el enfermero a través de su personalidad y actitud, guía los resultados de aprendizaje durante 
el período en que se prestan los cuidados. Este proceso interpersonal es un instrumento educativo, una fuerza que ayuda a madurar y que se propone facilitar una vida en toda su plenitud. Cabe resaltar, que el formar profesionales de la salud implica asumir el reto de crear y desarrollar una mente inquisitiva, preparada para estar en un permanente cuestionamiento, es decir, consolidar investigadores, que desde diferentes disciplinas, aporten al conocimiento colectivo de la sociedad, Rodríguez (2013). Para esto, es básico que se moldee una actitud positiva hacia la generación del conocimiento y la ciencia. La práctica de ciencias médicas, como enfermería, se establece generalmente a erradicar las alteraciones que afectan la salud del individuo, es decir, la enfermedad; pudiendo ser psicológica o fisiológica.

No obstante, se olvida así al hombre como ser social y el marcado carácter humano que debe prevalecer en la atención médica; esta y otras observaciones, llevan a reflexionar sobre la visión axiológica de las ciencias de la salud, por lo que el presente estudio está encaminado a investigar el enfoque axiológico y destacar el carácter valorativo de la ciencia de la salud, en este caso, enfermería; partiendo de conceptos elementales y del perfil humanista de esta ciencia. Por ende, más allá de esto poder enfatizar el pensamiento valorativo en el profesional de enfermería, a partir de la formación de valores en educandos, futuros profesionales capaces de actuar y evaluar una situación determinada del paciente, sustentados en los principios humanos y éticos. Formalizando una estrategia como herramienta en los cuidados que se le brindan al usuario, donde los valores prevalezcan, la sabiduría, confidencialidad y la bioética como fundamento a salvaguardar la vida de todo ser, que se atienda.

\subsection{El espacio como discurso formativo para el profesional de enfermería}

El espacio como aula de clases universitaria siempre ha sido pretendida como un tipo de ámbito humano en el que convergen personas que asumen 
característicamente dos tipos de roles: están quienes ejercen el papel de participantes de un proceso de adquisición y/o construcción de conocimientos de orden académico, personal y social, que frecuentemente deben mostrar competencias en el aprendizaje de tales conocimientos (estudiantes); por otra parte, están quienes tienen la responsabilidad de guiar, mediar o facilitar dicho proceso académico, empleando para ello una perspectiva de aprendizaje conforme a un modelo teórico particular y un modelo andragógico, que a su vez está conexo al conductismo, cognitivismo, humanismo y la teoría actual el constructivismo, (docentes).

Conforme, a la experiencia formal de estrategias de enseñanza aprendizaje, en la que participan tanto docentes como estudiantes, el aula de clases debe ser también vista como un hábitat con una ecología particular, en la que el éxito del proceso académico depende menos del fundamento psicológico/pedagógico que adopte el/la docente y más de las condiciones para el intercambio y la cooperación entre las personas. En efecto, el espacio como situación geográfica de un lugar, busca demostrar en este caso la interpretación de incentivos formativos, según Terán (2016a:85), "el espacio como discurso del silencio que se manifiesta en la producción de significados y significantes del hecho educativo, el cual forma el pensamiento del otro". Esto quiere decir, que el espacio es todo centro de concentración para un dicho fin, como lo es la formación de conocimientos.

Por consiguiente, los actores del proceso formativo deben estar atentos y a la vanguardia, así como comprender que todo lo que rodea al ser humano es porta voz de discursos, por tal motivo, el espacio universitario es un macro mundo lleno de un montón de significantes que demuestran la trayectoria de cada estudiante y docente que evoluciona o se dispersa de ese entorno; como ámbito físico, educativo, afectivo, entre otros. El espacio es un agitador de pensamiento, pues causa alteración o armonía en quien lo habita y de tal modo influye en la corriente o ideología de su interés. Reseña Terán (2016b:87), el 
espacio en el contexto donde el "tú", el "yo" y el "nosotros" convergen. Ese lugar donde un colectivo - individual se forma, intercambia pensamientos, conocimientos y saberes con todos los que conviven en él.

Ese departo en el escenario de estudio como espacio, produce una perspectiva, donde una serie de símbolos e imágenes se ven representadas en las paredes, puertas y todos los elementos que allí se presentan, con un fin el mostrar su origen, misión, visión, objetivos, lideres, y muchos más. Por tal razón, el mundo llamado "universidad", posee una carga semántica llena de elementos, tanto naturales como artificiales, espacios teñidos de colores verdes y cielos azules como espacios blancos, cargados de "grafitis" que muestran una estética del caos, del poco sentido de identidad y pertenencia de quienes le habitan.

\section{Referencias}

Ibáñez, T. (2005). Elementos críticos de la hermenéutica. México: Editorial Siglo XXI.

Martínez, V., \& Pérez, O. (s.f.). Semiótica del discurso desde el aula. Barcelona, España: CCS.

Mercer, N. (1996). Las perspectivas socioculturales y el estudio del discurso en el aula. En Coll, C., \& Edwards, D. (eds.), Enseñanza, aprendizaje y discurso en el aula. Aproximaciones al estudio del discurso educacional (11-21). Madrid, España: Fundación Infancia y Aprendizaje.

Morín, E. (1999). La cabeza bien puesta. Repensar la reforma. Reformar el pensamiento. Buenos Aires, Argentina: Ediciones Nueva Visión.

Rodríguez, J. (2013). Aptitud para la lectura crítica de trabajos de investigación clínica en residentes de cirugía general. México: Ediciones Modernidad.

Ricoeur, P. (1995). La metáfora viva. Madrid, España: Ediciones Europa. 
Terán, C. (2016a,b). Aproximaciones teóricas al discurso docente en la formación de estudiantes de educación. Tesis Doctoral. Venezuela: Universidad Nacional Experimental "Rafael María Baralt".

Van Dijk, T. (1999a,b). El análisis crítico del discurso. Anthropos (Barcelona), 186, págs. 23-36. Recuperado de:

http://www.discursos.org/oldarticles/El\%20an\%E1lisis\%20cr\%EDtico\% 20del\%20discurso.pdf 


\section{Carol del Carmen Terán González}

e-mail: carolteranula@gmail.com

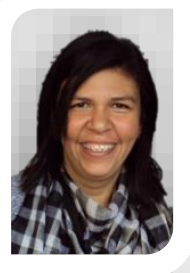

Nacida en la ciudad de Valera Estado Trujillo, Venezuela. Curso estudios de Doctorado en Educación en la Universidad Rafael María Baralt, posee Maestría en Docencia para la Educación Superior en la misma universidad, Maestría en Literatura Latinoamericana en la Universidad de los Andes y Licenciatura en Educación Mención Castellano y Literatura ULA-NURR investigadora del Centro de investigaciones literarias y lingüísticas Mario Briceño Iragorry. (CILL) ULANURR, Jefe de la unidad de investigación y proyecto de la Casa de Historia Trujillo, articulista e investigadora con 14 años de servicio en el área de educación y literatura. Ponente en eventos nacionales e internacionales. Docente invitada en pregrado y Postgrado, ULA, UNERMB, UNESR. Coordinadora de la Línea de investigación Memoria, Educación y Discursos emergentes (UNERMB), miembro de la Línea de investigaciones Ciudadanía, hermenéutica y proyectos sociales. (UNERS). Ha realizado artículos en distintas revistas arbitradas del país entre ella Sapiencia, Cifra Nueva, Revista de Cultura Centro Nacional de Historia, Revista de Ingeniería UVM, entre otras. 


\section{Yurquelis Rico Chacón \\ e-mail: yurquelisricoch@gmail.com}

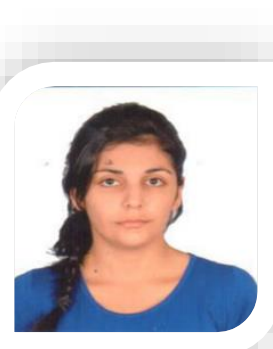

Nació en San Juan de Colon Estado Táchira, Venezuela. Estudio técnico superior en enfermería en el Instituto Universitario Gran Colombia en San Cristóbal, Edo. Táchira, Licenciatura en enfermería en la Universidad de los Andes en Valera Edo. Trujillo, Maestrante de Docencia para la Educación Superior en la Universidad Nacional Experimental Rafael María Baralt, labora como docente Universitaria en la Universidad Nacional Experimental de las fuerzas armadas, línea de investigación Memoria, educación y discursos emergentes. UNERMB.

El contenido de este manuscrito se difunde bajo una Licencia de Creative Commons Reconocimiento- 